\title{
Acute renal failure in COVID-19 patients on mechanical ventilation
}

\author{
Insuficiencia renal aguda en pacientes COVID-19 \\ en ventilación mecánica
}

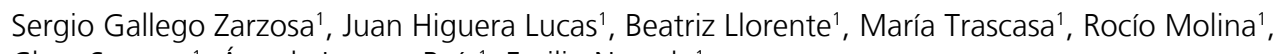
Clara Serranoํ, Ángela Leonor Ruíz', Emilio Nevado

\section{ABSTRACT}

Introduction: COVID-19 Community acquired pneumonia is a recent and frequent cause of admission in European intensive care units. Currently, there are many open questions regarding the management and prognostic factors of these patients. Among them, its association with acute renal failure in patients on mechanical ventilation. Objetive: Analysis of acute renal failure in COVID-19 patients on mechanical ventilation. Prognosis and factors related to its development. Material and Methods: Retrospective observational study carried out in the intensive care unit of a University Hospital during the COVID-19 pandemic. We analysed patients admitted to the ICU with the diagnosis of Respiratory Failure due to Pneumonia COVID-19 between 6/3/2020 and 21/4/2020. Demographic data (age, sex), APACHE II, comorbidities, analytical data (ferritin, total bilirubin), length of stay, treatments administered (mechanical ventilation, muscle relaxant, vasoactive drugs, prone) and its association to acute renal failure were analysed. Results: 67 patients received complete treatment in our hospital. Length of stay (days) $14.1 \pm 10$, average age 60 years, APACHE II $14.3 \pm 5.2,67.2 \%$ males. Maximum creatinine mean value $(\mathrm{mg} / \mathrm{dl}) 1.48 \pm 1.26$, Maximum ferritin mean value $(\mathrm{ng} / \mathrm{ml}) 2,310 \pm 3,322$, Maximum total bilirubin mean value $(\mathrm{mg} / \mathrm{dl}) 2 \pm 2.2$. $100 \%$ of the patients required mechanical ventilation. $65.7 \%$ prone positioning, $74.6 \%$ neuromuscular blockers and $98.5 \%$ required vasoactive drugs. Establishing renal failure as creatinine values greater than $1.2 \mathrm{mg} / \mathrm{dl}$. Patients with maximum creatinine above $1.2 \mathrm{mg} / \mathrm{dl}$ presented average values of bilirubin and ferritin higher than those with values under $1.2 \mathrm{mg} / \mathrm{dl}(P<0.05)$. The presence of renal failure was also statistically significantly associated with sex (male), presence of dyslipidaemia, and mortality. Conclusions: In our sample, we have associated acute renal
\end{abstract}

\section{Key words:}

Acute renal failure, COVID-19, mechanical ventilation, intensive care

Servicio de Medicina Intensiva del Hospital Príncipe de Asturias. Madrid, España.

Fecha de recepción: 01 de septiembre de 2020

Fecha de aceptación :12 de septiembre de 2020

\section{ORCID}

https://orcid.org/0000-0003-1778-2291

Correspondencia:

Juan Higuera Lucas

jhiguera.151@gmail.com 
failure with higher ferritin values. Likewise, we have observed higher creatinine values in the group of non-survivors, those with dyslipidaemia and men, with statistical signification.

\section{RESUMEN}

Introducción: La neumonía adquirida en la comunidad COVID-19 es una causa reciente y frecuente de ingreso en las unidades de cuidados intensivos europeos. Actualmente, hay muchas preguntas abiertas con respecto al manejo y los factores pronósticos de estos pacientes. Entre ellos, su asociación con insuficiencia renal aguda en pacientes en ventilación mecánica. Objetivo: Análisis de insuficiencia renal aguda en pacientes con COVID-19 en ventilación mecánica. Pronóstico y factores relacionados con su desarrollo. Material y Métodos: Estudio observacional retrospectivo realizado en la unidad de cuidados intensivos de un Hospital Universitario durante la pandemia de COVID-19. Analizamos los pacientes ingresados en $\mathrm{UCl}$ con el diagnóstico de insuficiencia respiratoria por neumonía COVID-19 entre el 3/6/2020 y el 21/4/2020. Se analizaron datos demográficos (edad, sexo), APACHE II, comorbilidades, datos analíticos (ferritina, bilirrubina total), tiempo de estancia, tratamientos administrados (ventilación mecánica, relajante muscular, fármacos vasoactivos, prono) y su asociación con insuficiencia renal aguda. Resultados: 67 pacientes recibieron tratamiento completo en nuestro hospital. Duración de la estancia (días) 14,1 \pm 10 , edad media 60 años, APACHE II 14,3 \pm 5,2, 67,2\% varones. Valor medio máximo de creatinina $(\mathrm{mg} / \mathrm{dl}) 1,48 \pm 1,26$, valor medio máximo de ferritina $(\mathrm{ng} / \mathrm{ml}) 2.310$ \pm 3.322 , valor medio máximo de bilirrubina total $(\mathrm{mg} / \mathrm{dl}) 2 \pm 2,2$. El $100 \%$ de los pacientes requirió ventilación mecánica. El 65,7\% en decúbito prono, el $74,6 \%$ bloqueantes neuromusculares y el 98,5\% requirieron fármacos vasoactivos. Estableciendo la insuficiencia renal como valores de creatinina superiores a 1,2 mg/dl, los pacientes con creatinina máxima superior a 1,2 mg/dl presentaron valores medios de bilirrubina y ferritina superiores a aquellos con valores menores a 1,2 mg/dl, de forma estadísticamente significativa. La presencia de fallo renal, también se asoció de forma estadísticamente significativa al sexo hombre, la presencia de dislipidemia como antecedente personal de interés y a la mortalidad. Conclusiones: En nuestra muestra hemos asociado la insuficiencia renal aguda con valores más elevados de ferritina. Asimismo, hemos observado mayores valores de creatinina en el grupo de no supervivientes, los que presentan dislipidemia y los hombres, con significación estadística.

\section{Palabras clave:}

Insuficiencia renal aguda,

COVID-19, ventilación mecánica, cuidados intensivos

\section{Introducción}

a situación emergente global que hemos vivido a causa de la enfermedad por el coronavirus SARSCoV-2 (Severe acute respiratory syndrome-coronavirus-2) ha acelerado la necesidad de identificar los valores de laboratorio que se puedan asociar a peor pronóstico. Esto, además, podría suponer un mayor conocimiento de la fisiopatología de la enfermedad y, por tanto, una mejor aproximación a unas medidas preventivas y terapéuticas eficaces.
El porcentaje de casos severos es escaso en relación a los asintomáticos o leves. Sin embargo, el SARS-CoV-2 no sólo se limita a producir distress respiratorio, sino que tiene potencial para afectar a todos los sistemas, incluso en pacientes sin comorbilidad previa. La experiencia nos muestra que, además, puede provocar encefalopatía, trastornos de la coagulación, miocarditis, fallo hepático y fallo renal, empeorando hasta diez veces el pronóstico del paciente[1].

Diversos estudios han establecido diferencias sig- 
nificativas en los hallazgos analíticos en los grupos de pacientes con enfermedad severa, y no severa, encontrando en general que, aunque la mayoría de los pacientes poseen un patrón determinado de marcadores inflamatorios, hematológicos, bioquímicos e inmunológicos, no está igual de alterado en ambas poblaciones[2]. Nuestro interés en el presente estudio es analizar qué resultados de laboratorio se encuentran asociados a mayor mortalidad en el grupo de pacientes con enfermedad severa, especialmente su asociación con el fallo renal agudo en estos pacientes.

\section{Material y Métodos}

Se realiza un estudio retrospectivo, observacional, con todos los enfermos que ingresan en el Servicio de Medicina Intensiva en un Hospital Universitario con el diagnóstico clínico o analítico de neumonía adquirida en la comunidad (NAC) grave COVID-19. El estudio cuenta con la aprobación del comité de ética del Hospital Universitario Príncipe de Asturias. Los pacientes analizados fueron aquellos que ingresaron en la unidad de cuidados intensivos desde el 6/3/2020 al 21/4/2020. En el análisis completo del estudio, analizamos los pacientes que recibieron tratamiento completo en nuestro hospital, excluyendo los traslados a otros centros hospitalarios.

Se define neumonía adquirida en la comunidad (NAC) según los criterios de las guías internacionales referentes a esta patología y grave cuando cumplen criterios de ingreso en $\mathrm{UCl}$ por insuficiencia respiratoria o fallo hemodinámico. En todos los pacientes incluidos en este estudio el motivo de ingreso fue insuficiencia respiratoria aguda grave con necesidad de ventilación mecánica invasiva. El diagnóstico de neumonía por COVID-19 se hace en base a un patrón radiológico característico y prueba PCR positiva para COVID-19. El primer ingreso en nuestra Unidad por neumonía adquirida en la comunidad grave COVID-19 fue el 06/03/2020.

Se recogieron las siguientes variables: edad, sexo, principales comorbilidades (diabetes mellitus, hipertensión arterial, enfermedad pulmonar previa, patología cardiovascular y otros), índice de gravedad (APACHE II), presencia de fallo orgánico, valores bioquímicos (creatinina, urea, ferritina) ventilación en prono, necesidad de relajantes neuromusculares, días de estancia en $\mathrm{UCl}$ y mortalidad intraUCl. La ventilación en prono se llevó a cabo en pacientes con presión arterial de $\mathrm{O}_{2} /$ Fracción inspiratoria de oxígeno $\left(\mathrm{PaFiO}_{2}\right)$ $<120$. El uso de relajantes neuromusculares se llevó a cabo en pacientes con presión arterial de $\mathrm{O}_{2} /$ Fracción inspiratoria de oxígeno $\left(\mathrm{PaFiO}_{2}\right)<150$. En el análisis del estudio hemos establecido el valor de creatinina mayor de 1,2 mg/dl como fallo renal agudo.

Se realiza el análisis estadístico mediante la herramienta IBM ${ }^{\circledR S P S S}$ Statistics 23 . La normalidad de las variables ha sido establecida mediante el test de Komogorov-Smirnov. Las variables cuantitativas con distribución normal han sido expresadas como media \pm desviación estándar (rango). Estas, han sido comparadas mediante test t-Student o ANOVA. Las distribuciones cuantitativas que no seguían una distribución normal han sido comparadas utilizando Test de Wilcoxon y son expresadas como mediana y rango intercuartílico. Las variables cualitativas son mostradas como números y porcentajes. Son comparadas mediante test de McNemar y Chi-cuadrado. El nivel de significación estadística se ha establecido con valores de $\mathrm{P}$ menores de 0,05 . Los resultados son expresados como intervalos de confianza del 95\%.

\section{Resultados}

Se analizan un total de 90 pacientes. Todos los pacientes ingresan en la unidad de cuidados intensivos por insuficiencia respiratoria aguda hipoxémica diagnosticados de neumonía de comunidad COVID-19 requiriendo ventilación mecánica. Los pacientes analizados han sido ingresados, trasladados a otros centros hospitalarios de apoyo por falta de camas, dados de alta o han fallecido. Para realizar el presente análisis, excluimos los pacientes trasladados, seleccionando únicamente los que recibieron terapia completa en nuestro hospital (67). El 44,8\% (30/67) de los pacientes cuentan con antecedentes previos cardiovasculares siendo la hipertensión arterial el diagnóstico más frecuente. El 19,4\% (13/67) de los pacientes presentaban antecedentes pulmonares crónicos. El $62,7 \%$ de los pacientes presentaban otro tipo de comorbilidad, siendo la dislipidemia y diabetes mellitus las más frecuentes. El 19,4\% (13/67) no contaba con ninguna enfermedad previa a su ingreso en medicina intensiva. Ni la presencia de antecedentes personales importantes, ni su ausencia se relacionaron significativamente con mayor incidencia de traqueotomía, pronación, relajación, fármacos vasoactivos, fallo renal o hepático y estancia media (Tabla 1).

Los valores de ferritina media no se relacionaron de forma significativa a la presencia de antecedentes personales importantes, pero la dislipidemia sí se asoció a peor APACHE II de ingreso 17,1 vs $12,3 \%(p=0,002)$; a peores cifras de creatinina 1,9 vs $1,22(p=0,02)$; y a mayor necesidad de prono $80 \%$ vs $50 \%(p=0,04)$. 


\begin{tabular}{|c|c|c|c|c|c|c|c|c|c|c|c|c|}
\hline & \multicolumn{3}{|c|}{ CV } & \multicolumn{3}{|c|}{ Pulmonares } & \multicolumn{3}{|c|}{ Sin Ap. } & \multicolumn{3}{|c|}{ DL } \\
\hline & Sí & No & $\mathbf{P}$ & Sí & No & $\mathbf{P}$ & Sí & No & $\mathbf{P}$ & Sí & No & $\mathbf{P}$ \\
\hline & 30 & 37 & & 13 & 54 & & 13 & 54 & & 25 & 42 & \\
\hline $\begin{array}{l}\text { Sexo } \\
\text { (Hombre) }\end{array}$ & $76,6 \%$ & $59,4 \%$ & 0,1 & $76,9 \%$ & $64,7 \%$ & 0,3 & $76,9 \%$ & $64,8 \%$ & 0,3 & $64 \%$ & $69 \%$ & 0,43 \\
\hline Apache II & 14,7 & 14 & 0,68 & 14,3 & 14,3 & 0,9 & 11,89 & 14,9 & 0,125 & 17,1 & 12,35 & 0,002 \\
\hline Ferritina & 1.526 & 2.856 & 0,14 & 2.440 & 2.285 & 0,9 & 3.000 & 2.100 & 0,4 & 2.156 & 2.389 & 0,8 \\
\hline Creatinina & 1,69 & 1,31 & 0,2 & 1,2 & 1,55 & 0,37 & 1,3 & 1,5 & 0,6 & 1,9 & 1,22 & 0,02 \\
\hline Bilirrubina & 2,27 & 1,8 & 0,4 & 1,6 & 2,1 & 0,48 & 2,5 & 1,9 & 0,47 & 2,65 & 1,6 & 0,07 \\
\hline Prono & $66,6 \%$ & $64 \%$ & 0,34 & $53,8 \%$ & $68 \%$ & 0,24 & $60 \%$ & $66 \%$ & 0,48 & $80 \%$ & $57 \%$ & 0,04 \\
\hline $\begin{array}{l}\text { Traqueosto- } \\
\text { mía }\end{array}$ & $50 \%$ & $41,6 \%$ & 0,34 & $50 \%$ & $44 \%$ & 0,5 & $30 \%$ & $50 \%$ & 0,19 & $43,4 \%$ & $46,6 \%$ & 0,5 \\
\hline $\begin{array}{l}\text { Estancia } \\
\text { media }\end{array}$ & 13,6 & 14,6 & 0,67 & 11,2 & 14,9 & 0,2 & 13 & 14,4 & 0,6 & 15 & 13,5 & 0,6 \\
\hline
\end{tabular}

Excluyendo los pacientes trasladados, analizando los 67 pacientes que recibieron tratamiento completo en nuestro hospital: los días medios de ingreso fueron 14,1 \pm 10 , edad media 60 años, APACHE II medio $14,3 \pm 5,2.67,2 \%$ varones. Valor medio de peor resultado de creatinina $1,48 \pm 1,26$, valor medio de peor valor de ferritina $2.310 \pm 3.322$, valor medio de peor valor de bilirrubina total $2 \pm 2,2$. El $100 \%$ de los pacientes requirieron ventilación mecánica. El 65,7\% ciclos de pronación, el 74,6\% perfusión de relajantes y el $98,5 \%$ fármacos vasoactivos. En $45,3 \%$ de los pacientes se realizó traqueotomía. En la Tabla 2 se muestran las principales características dividiendo estos pacientes en supervivientes y no supervivientes.

Estableciendo la insuficiencia renal como valores de creatinina superiores a $1,2 \mathrm{mg} / \mathrm{dl}$, se obtuvieron los resultados que se muestran en la Tabla 3. En la Tabla 4 se muestran los valores medios de creatinina en pacientes que requieren decúbito prono, bloqueadores musculares, enfermedad cardiovascular, dislipidemia, supervivientes y no supervivientes.

\section{Discusión}

Aunque la incidencia de fallo renal asociado a la COVID-19 es baja (en torno al 6\%), sí es preocupante por la elevada mortalidad, hasta 94\%[3]. Según series, la incidencia de fallo renal varía de $1,3 \%$ al $36,4 \%$, dependiendo de la severidad de la enfermedad. Asimismo, la incidencia de AKI puede ser hasta 5 veces superior en los casos severos que en los no severos o supervivientes[4].

En nuestra muestra se sigue esta misma tendencia, con mayor mortalidad entre los pacientes con cifras de creatinina elevadas, y mayor creatinina máxima en el grupo de los no supervivientes, de manera estadísticamente significativa.

El riñón se ve afectado por acción directa del propio virus, pero además, existen otros mecanismos que contribuyen al daño renal, como la hipoxia o la hipercoagulabilidad. En una serie de autopsias realizadas a 26 pacientes adultos infectados por coronavirus, no sólo se encontraron datos de infección parenquimatosa directa, sino también agregados eritrocitarios en el lumen de los capilares glomerulares y peritubulares sin plaquetas, esquistocitos o trombos de fibrina $y$, además, se encontraron datos de rabdomiolisis[5].

Probablemente, un trastorno endotelial previo ponga en especial riesgo la circulación renal y condicione mayor tasa de fallo del órgano, ya que, aunque no hayamos podido establecer de manera significativa la relación entre enfermedad cardiovascular previa con mayores niveles de creatinina, sí hemos podido relacionar la dislipidemia con esto. Además, se ha demostrado que el propio virus es capaz de producir endotelitis, ya que el receptor ACE2, al que se adhiere[6], también se expresa en las células endoteliales[7]. Se han hallado inclusiones víricas en las células endoteliales y acúmulo de células inflamatorias en este tejido, lo cual lleva a disfunción endotelial, vasoconstricción y la subsiguiente isquemia del órgano[7]. La elevación encontrada en la literatura previa de biomarcadores como la IL-6 o la ferritina sugieren un 


\begin{tabular}{|c|c|c|c|}
\hline & Supervivientes & No supervivientes & $\mathbf{P}$ \\
\hline Edad & 56,9 & 63,4 & 0,006 \\
\hline $\operatorname{Sexo}(H)$ & $55 \%$ & $80 \%$ & 0,026 \\
\hline APACHE ॥ & 12,6 & 16 & 0,03 \\
\hline Estancia UCI & 16,5 & 11,5 & 0,042 \\
\hline Creatinina máx. & 1 & 1,96 & 0,003 \\
\hline Bilirrubina máx. & 1,71 & 2,4 & 0,2 \\
\hline Ferritina máx. & 1.836 & 2.990 & 0,2 \\
\hline D Dímero & 17,2 & 19,9 & 0,67 \\
\hline AP CV & $38,8 \%$ & $51 \%$ & 0,21 \\
\hline AP pulmonar & $19,4 \%$ & $19,3 \%$ & 0,6 \\
\hline Dislipidemia & $27,7 \%$ & $48 \%$ & 0,069 \\
\hline Traqueostomía & $51 \%$ & $37,4 \%$ & 0,2 \\
\hline Prono & $52,7 \%$ & $80 \%$ & 0,015 \\
\hline Relajación neuromuscular & $58 \%$ & $93 \%$ & 0,001 \\
\hline
\end{tabular}

Tabla 3. Valores medios o máximos de edad, APACHE II, ferritina, dímero-D, bilirrubina en los pacientes con creatininas mayores o menores de $1,2 \mathrm{mg} / \mathrm{dl}$

\begin{tabular}{lccc}
\hline & Creatinina $>\mathbf{1 , 2} \mathbf{~ m g / d l}$ & Creatinina $<\mathbf{1 , 2} \mathbf{~ m g / d l}$ & $\mathbf{P}$ \\
Edad (años) & 61,3 & 59 & 0,37 \\
APACHE II & 15,8 & 13,3 & 0,128 \\
Ferritina (máx) & 3.421 & 1.535 & 0,035 \\
Dímero-D (máx) & 23,5 & 15 & 0,19 \\
Bilirrubina (máx) & 3 & 1,4 & 0,012 \\
\hline
\end{tabular}

\begin{tabular}{|c|c|c|c|}
\hline & $\begin{array}{l}\text { Valor medio de creatinina } \\
\text { (mg/dl) }\end{array}$ & & $\mathbf{P}$ \\
\hline & Sí & No & \\
\hline Prono & 1,51 & 1,43 & 0,8 \\
\hline Relajación muscular & 1,49 & 1,46 & 0,9 \\
\hline $\operatorname{Sexo}(H)$ & 1,81 & 0,8 & 0,002 \\
\hline Enfermedad CV & 1,69 & 1,3 & 0,22 \\
\hline Dislipidemia & 1,92 & 1,22 & 0,026 \\
\hline Sobreinfección & 1,62 & 1,3 & 0,29 \\
\hline Supervivientes & 1,07 & 1,96 & 0,003 \\
\hline
\end{tabular}


estado hiperinflamatorio. La elevación de la ferritina se asocia con mayor mortalidad en la sepsis y fracaso multiorgánico, lo que también parece suceder en el caso de pacientes con enfermedad severa por SARSCoV-2. Respecto a esto, se ha sugerido que la ferritina no solo es consecuencia de la tormenta inflamatoria, sino que podría tomar parte activa perpetuando y amplificando la respuesta inflamatoria sistémica, ya que la ferritina toma papel protagonista en los síndromes hiperferritinémicos (síndrome de activación macrofágica, enfermedad de Still, síndrome antifosfolípido y shock séptico). La COVID-19 comparte características con éstos, y probablemente forme parte del mismo espectro clínico[8].

De esta manera, aunque en nuestra muestra no hallamos que la hiperferritinemia se asocie por sí sola a mayor mortalidad de manera estadísticamente significativa, sí se asocia a mayor incidencia de insuficiencia renal.

La ferritina es un marcador inflamatorio directamente relacionado con el síndrome de respuesta inflamatoria sistémica (SIRS)[9], encontrándose elevada en los pacientes con enfermedad severa. Esta molécula se libera tras un estímulo proinflamatorio, que no sólo modifica la disponibilidad extracelular de hierro, sino que regula un mecanismo independiente del hierro, que activa citoquinas proinflamatorias que se suman a las activadas por la COVID-19, resultando en un círculo vicioso de activación inflamatoria[10]. Esto también afecta a la coagulación, tanto activándola como disminuyendo los mecanismos compensatorios, provocando un estado procoagulante, el cual va a afectar a diversos órganos, sobre todo pulmón, cerebro y riñón.

Todas estas alteraciones de laboratorio llevan a lo mismo: alteración endotelial, estado procoagulante y, finalmente, afectación por diversos mecanismos de la nefrona, que va a llevar a la pérdida del órgano. Nuestros datos asocian claramente el fallo renal con la mortalidad, y la hiperferritinemia con el fallo renal.

Se trata de un estudio pequeño y retrospectivo, por lo que cuenta con limitaciones, pero añade evidencia y refuerza la evidencia clínica que de momento se está estableciendo en el resto de la literatura.

\section{Conclusión}

El fracaso renal y la dislipidemia se asocian a mayor mortalidad. Una de las posibles explicaciones, teniendo en cuenta la elevación de otros reactantes de fase aguda como la ferritina nos sugiere que el daño endotelial podría ser la causa de este fallo de órgano. Se precisan, no obstante, más estudios al respecto.

\section{Referencias}

1. Lim MA, Pranata R, Huang I, Yonas E, Soeroto AY, Supriyadi R. Multiorgan Failure With Emphasis on Acute Kidney Injury and Severity of COVID-19: Systematic Review and Meta-Analysis. Can J Kidney Health Dis. 2020 Jul; $7: 2054358120938573$. https://doi. rg/10.1177/2054358120938573 PMID:32685180

2. Henry BM, de Oliveira MHS, Benoit S, Plebani M, Lippi G. Hematologic, biochemical and immune biomarker abnormalities associated with severe illness and mortality in coronavirus disease 2019 (COVID-19): a metaanalysis. Clinical Chemistry and Laboratory Medicine (CCLM). 25 de junio de 2020;58(7):1021-8. https://doi.org/10.1515/cclm2020-0369

3. Bajwa H, Riaz Y, Ammar M, Faroog S, Yousaf A. The Dilemma of Renal Involvement in COVID-19: A Systematic Review. Cureus. 15 de junio de 2020;12(6):e8632. https://doi. org/10.7759/cureus.8632

4. Yang $X$, Jin $Y$, Li R, Zhang Z, Sun $R$, Chen D. Prevalence and impact of acute renal impairment on COVID-19: a systematic review and metaanalysis. Crit Care. diciembre de 2020;24(1):356. https:// doi.org/10.1186/s13054-02003065-4

5. Su H, Yang $M$, Wan C, Yi L-X, Tang F, Zhu H-Y, et al. Renal histopathological analysis of
26 postmortem findings of patients with COVID-19 in China. Kidney International. julio de 2020;98(1):219-27. https://doi. org/10.1016/j.kint.2020.04.003

6. Driggin E, Madhavan MV, Bikdeli B, Chuich T, Laracy J, BiondiZoccai G, et al. Cardiovascular Considerations for Patients, Health Care Workers, and Health Systems During the COVID-19 Pandemic. Journal of the American College of Cardiology. mayo de 2020;75(18):235271. https://doi.org/10.1016/j. jacc.2020.03.031

7. Varga Z, Flammer AJ, Steiger $P$, Haberecker M, Andermatt R, Zinkernagel AS, et al. Endothelial cell infection and endotheliitis in COVID-19. The Lancet. mayo de 2020;395(10234):1417-8. 
https://doi.org/10.1016/S01406736(20)30937-5

8. Colafrancesco $S$, Alessandri C, Conti F, Priori R. COVID-19 gone bad: A new character in the spectrum of the hyperferritinemic syndrome? Autoimmunity Reviews. julio de 2020;19(7):102573. https://doi.org/10.1016/j.au- trev.2020.102573

9. Giemza-Stokłosa J, Islam MA, Kotyla PJ. Hyperferritinaemia: An Iron Sword of Autoimmunity. Curr Pharm Des. 2019;25(27):2909-18. https://doi.org/10.2174/138 1612825666190709202804 PMID:31686632

10. Ruscitti P, Berardicurti O, Di
Benedetto $P$, Cipriani $P$, lagnocco $A$, Shoenfeld $Y$, et al. Severe COVID-19, Another Piece in the Puzzle of the Hyperferritinemic Syndrome. An Immunomodulatory Perspective to Alleviate the Storm. Front Immunol. 28 de mayo de 2020;11:1130. https://doi.org/10.3389/ fimmu.2020.01130 